\title{
Le réseau de médecins idéal
}

Le réseau de médecins de la région de Thoune, IGOMED, a été fondé en 1996 et compte parmi les plus anciens et les plus étendus en Suisse. Sa particularité: les spécialistes régionaux et l'hôpital de Thoune y sont associés. L'IGOMED compte actuellement 162 membres (115 médecins de premier recours, 47 spécialistes) et plus de 22000 patients, avec un modèle d'assurance Managed care.

Peter Bachmann,

Ueli Bodmer, Andreas Frenzer, Ueli Krebs, Paul Mäder, Mani, Hans Jakob Zehnder

Comité et direction du réseau IGOMED

\section{- JIGOMED \\ ÄRZTENETZWERK RAUM THUN} Theo Rohrer, Ulrich Weber-

La controverse sur le MC révèle une fois de plus le fossé au sein du corps médical, de part et d'autre du rideau de rösti. Ces orientations politiques de long terme, qui ont des conséquences sur tout le secteur ambulatoire, trahissent intrinsèquement l'hétérogénéité des personnes concernées.

Le modèle MC contient de nombreux éléments souhaitables: on ne peut contester l'optimisation de la chaîne de soins, la suppression des procédures en double, le respect des critères WZW (Wirksamkeit/ Zweckmässigkeit/Wirtschaftlichkeit; efficacité/utilité/rentabilité), l'étroite mise en réseau des médecins, pour un suivi optimal du patient (soins intégrés), ni la nouvelle obligation contractuelle des assurances vis-à-vis des réseaux de médecin.

Pour les auteurs, le côté laborieux du référendum s'explique moins par l'aspect discutable de la compensation des risques (même si c'est le plus souvent le principal argument avancé, un compromis sera certainement trouvé) que par un point du modèle

\section{Le côté laborieux du référendum s'explique par un point du modèle $\mathrm{MC}$ autour duquel on joue avec les mots: le gatekeeping.}

Correspondance: Dr Peter Bachmann igomed[at]gmail.com
MC autour duquel on joue avec les mots: le gatekeeping. Le modèle est centré sur l'obligation imposée aux assurés de toujours consulter d'abord le généraliste qu'ils ont choisi. La plupart des réseaux MC actuels n'intégrant pas les spécialistes, et comme aucune base légale n'est envisagée à ce sujet, le généraliste devient le gatekeeper et parfois un lien incontournable entre le patient et le spécialiste.

Il revient donc au médecin de famille de décider où commence le «luxe» ou le «maximum», et où finit la médecine «raisonnable» ou «optimale» (termes des prises de position stratégiques du PLR et de medswiss.net). Cette décision devrait être basée sur des critères d'évidence (dans la mesure où il les connaît), mais elle dépend aussi de son niveau de formation, des points forts de sa formation personnelle, de ses préférences de travail, de ses intérêts et surtout de sa capacité à être collégial et à travailler en équipe.

On peut comprendre que cela suscite la réserve de nombreux spécialistes. Certains pensent même que le gatekeeping entraîne un risque de rationnement caché (dans l'intérêt des assurances) [1].

Cette position suppose que la médecine des spécialistes serait plus chère que celle des médecins de premier recours, ce qui est aussi absurde que d'imaginer que les médecins de premier recours feraient traîner leurs patients (risque d'augmentation des coûts) en retardant des prestations nécessaires. Ce type d'argument ne tient pas debout, ni d'un côté ni de l'autre.

Le gatekeeping du modèle MC actuel peut donc monter les médecins de premier recours contre les spécialistes, ce qui ne manquera pas d'être récupéré par les lobbyistes (diviser pour régner) et de compliquer les soins intégrés. Nous ne pouvons absolument pas nous permettre de désigner une discipline pour en contrôler une autre. Il ne viendrait à l'idée de personne d'inverser le discours et de déclarer les spécialistes comme gatekeepers, en leur attribuant le pouvoir de décider si un patient sera suivi au mieux entre les mains d'un médecin de premier recours.

La solution passe par une coopération optimisée (régulée) entre les médecins de premier recours et les spécialistes au sein d'un réseau MC, un peu sur le modèle du réseau de médecins IGOMED de Thoune, qui associe les spécialistes et l'hôpital.

Les principales caractéristiques du réseau IGOMED sont les suivantes:

- Les spécialistes régionaux et l'hôpital sont contractuellement associés au réseau.

- En adhérant à ce modèle de médecine de famille, les assurés ont la possibilité de consulter directement le spécialiste compétent en cas de problèmes spécifiques (sans passer par le médecin de famille). 


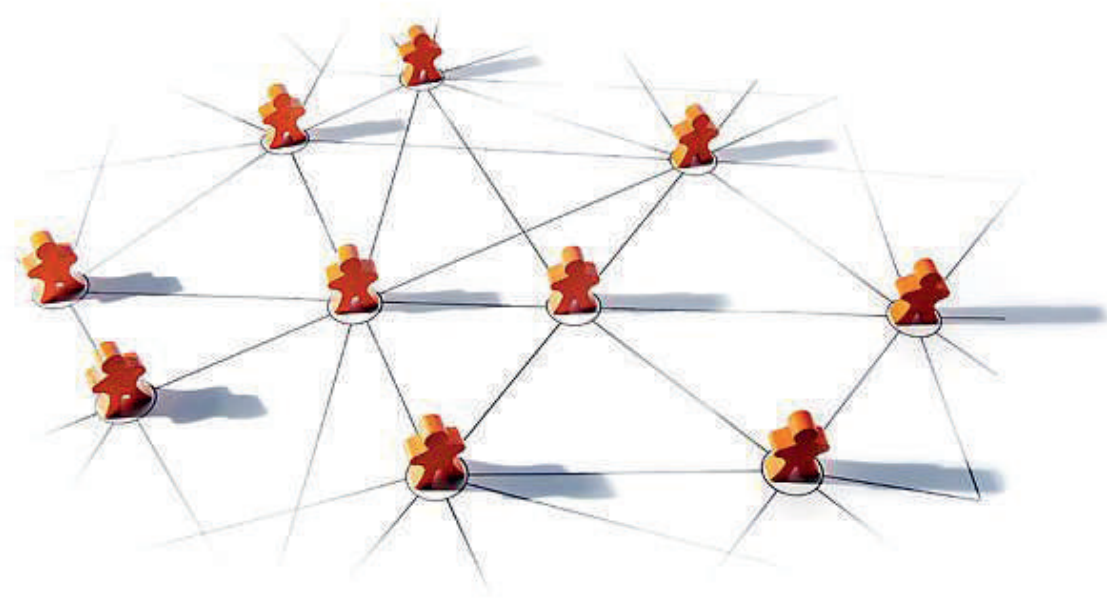

Dans un réseau de médecins on ne peut absolument pas permettre de désigner une discipline pour en contrôler une autre.
- Le réseau IGOMED a un concept de qualité obligatoire (formations continues internes au réseau, cercles de qualité, Critical Incident Report System/système de déclaration d'incidents critiques).

Visant une incitation supplémentaire à pratiquer une médecine qui respecte les critères WZW, le réseau IGOMED s'est accordé sur une CO-responsabilité budgétaire avec les caisses-maladie contractuellement liées à lui. Le réseau a accès aux données des assurances sur les coûts et peut poser des questions. Les objectifs d'économies sont renégociés tous les ans. Comme il s'agit d'une co-responsabilité, le réseau de médecins ne court aucun risque financier d'avoir un bilan négatif (remboursement aux assurances).

Comme il enregistre des bilans positifs et que son fonctionnement administratif est très léger, l'IGOMED est l'un des rares réseaux en mesure de dédommager financièrement les médecins de premier recours adhérents de leur implication (gestion des patients du réseau/gestion des coûts, cercles de qualité, etc.).

Depuis sa création, le réseau IGOMED fait des expériences exceptionnellement positives en matière de coopération de tous les médecins participants, hôpital compris, et de suivi de la chaîne de soins. Les procédures sont régulièrement améliorées, grâce entre autres à des groupes de travail ouverts à tous les participants. La coopération étant régie contractuellement et le réseau ayant un droit de regard sur les données concernant les coûts, le rapport entre réseau et assurances est empreint d'un plus grand respect (voire d'une véritable volonté de coopération de certaines assurances). Les négociations se font en direct et les décisions unilatérales appartiennent désormais à un passé révolu. De plus, cette structure de réseau est politiquement soutenue.

Le réseau IGOMED plaide clairement en faveur du Managed Care. Le modèle MC actuel constitue une nette amélioration par rapport à la loi existante. L'intégration des spécialistes, voire des hôpitaux, aux réseaux MC permet de lever les derniers obstacles importants. Une médecine judicieuse ne peut être pratiquée que si tous les participants coopèrent étroitement.

\section{Référence}

1 Nadig J. Unlautere Argumente der Managed-Care-Gegner. Bull Méd Suisses. 2011, 92(37):1410. Cela peut entraîner des conséquences.
Céné des côts supérieurs à la 